\title{
Zur Kenntnis der Micromycetenflora Finnlands 1. Für Finnland neue oder seltene Rost- und Mehltaupilze
}

Yrjö Mäkinen

Veröffentlichungen des Botanischen Institutes der Universität Turku, Nr. 45

Im Laufe mehreren Jahren habe ich u.a. auch Mikromyceten gesammelt. Weil die Flora Finnlands in bezug auf diese Pilze noch sehr mangelhaft - obschon besser als die vieler anderen Länder - bekannt ist, werde ich unter obigem Titel über einige wichtigere Funde berichten. Mit* sind die für Finnland neuen Arten, Wirtspflanzen und Sporenformen vermerkt. TUR $=$ Herbarium der Universität Turku. Die Grössenangaben der Sporen, Perithecien und Konidien gründen sich auf 100 Messungen (nach Erwärmung in Milchsäure).

\section{UREDINALES}

1. Coleosporium campanulae (Pers.) Lév. - Ab, Turku, Iso-Heikkilä, Botanischer Garten, 9.9.1953 Y.M., auf folgende Wirtspflanzen: Campanula rapunculoides L. (II + III, in Turku ganz allgemein), G. latifolia $\mathrm{L}$. (II). ${ }^{*}$ C. rapunculus $\mathrm{L}$. (II), ${ }^{*}$ G. bononiensis $\mathrm{L}$. (II), *C. steveni Bieb. (II), *C. sarmatica Ker-G. (II), *C. carpatica Jacq. (II). Die Infektion ist meistens verhältnismässig schwach, nur $C$. rapunculoides ist stark befallen.

2. Melampsora euphorbiae (Schub.) Cast. - Auf * Euphorbia palustris L.: Ab, Turku, Iso-Heikkilä, Botanischer Garten, 29.8.1953 Y.M. (I + II + III). Fast alle Blätter in dem etwa $1 \mathrm{~m}^{2}$ umfassenden dichten Bestand waren vom Pilz angegriffen. Caeomasporen $16-20 \times 18-21 \mu$, Uredosporen $11-14 \times$ 14-17 $\mu$ Paraphysen bis $65 \mu$ lang, keulenförmig mit bis $20 \mu$ dicker und $30 \mu$ langer Anschwellung. Junge Teleutosporen etwa $11 \times 45 \mu$. Das Material wurde als Nr. 634 in Fungi Exsiccati Fennici verteilt.

3. Phragmidium potentillae (Pers.) Karst. - Auf Potentilla thuringiaca Bernh. ssp. goldbachii (Rupr.) Adl. : Ta, Tampere, Vuohenoja, nahe der Haltestelle am Bahndamm, 6.8.1958 Y.M. (I + II). RAuHALA (1959, S. 85) sammelte diese Art schon i.J. 1952 nicht weit von der Stelle auf demselben Wirt, der hier an mehreren Punkten längs der Bahn vorkommt. Das Material wurde als Nr. 776 in Fungi Exsiccati Fennici verteilt.

4. Puccinia arenariae (Schum.) Wint. - Auf. *Stellaria alsine Grimm (S. uliginosa Murr.): Ta, Nokia, Haavisto, 30.8.1953 Y.M. (III). Teleutosporenlager nur an einigen Blättern, Sporen $14-17 \times 32-40 \mu$.

5. P. caricina DC. - Auf * Carex hirta L.: Ab. Uusikaupunki, Kause Oy, 16.8.1954 Y.M. (II + III) Teleutosporen $17-19 \times 50-63 \mu$.

6. P. chrysosplenii Grev. - Auf Chrysosplenium alternifolium L.: St, Hämeenkyrö, Osara, Äimälahti und St, Ikaalinen, Sikuri, Kaskenmäki, 28.8.1954 Y.M. (III). An beiden Stellen (etwa $700 \mathrm{~m}$ voneinander entfernt) trat der Pilz sehr spärlich und nur in den von Entyloma chrysosplenii verursachten Flecken auf. Die Wirtspflanze ist in der Gegend keineswegs selten, der Pilz wurde aber nur hier gefunden. In den obengenannten Proben sind die Sporen beinahe farblos, $10-15 \mu$ breit und $24-32 \mu$ lang. $P$. chrysosplenii ist in Finnland offenbar eine ziemlich grosse Seltenheit, 
für die auch LiRo (1908, S. 269) nur zwei Fundplätze in Ostfennoskandien anführt: N, Pornainen, Laha, 3.7.1903 J. I. Lindroth (spärlich) und Kol, Solomeno bei Petrosavodsk, 16.6.1898 J. I. Lindroth (sehr spärlich). Auch danach ist der Pilz meines Wissens bei uns nur einmal in $\mathrm{Ab}$ und dreimal in Ta gefunden worden (RAuHALA 1959, S. 101). Es verdient untersucht $\mathrm{zu}$ werden, ob er wirklich so selten ist, oder nur wegen seiner Unauffälligkeit unbemerkt geblieben ist.

7. P. coronata Corda - Auf *Avena fatua L.: Ab, Raisio, auf dem Bahnhof, 28.9.1953 Y.M. (II). Starker Befall, aber keine Teleutosporen. Die Uredosporen sind etwas grösser als bei $P$. coronata im allgemeinen, $24.1 \times 26.5 \quad(22-26 \times 24-29) \mu$, sehr hell, mit 4-5 Keimporen. Ab, Turku, Kanavaniemi, 10.10.1953 Y.M. (II + III).

8. P. dioicae P. Magn. - Auf* Carex praecox Schreb.: $\mathrm{N}$, »Ekenäs, ruderatmark invid Dragsviks hällplats», 12.7.1935 Olof Fortelius (II + III; TUR, Gefässpflanzenherbarium); Ta, Tampere, Santalahti, 25.5.1954 Y.M. (II). Uredosporen der ersteren Probe $18-21 \times 18-24 \mu$, der letzteren $18-22 \times$ $20-25 \mu$, die spärlichen Teleutosporen der ersteren etwa $16 \times 40 \mu$. Carex praecox als Wirtspflanze eines Rostpilzes ist neu für ganz Fennoskandien. - Auf * Carex disticha Huds.: Ab, Merimasku, Sannainen, 24.8.1923 Lauri E. Kari (II; TUR, Gefässpflanzenherbarium). Uredosporen $16-20 \times 20-24 \mu$. - Auf * Taraxacum officinale L., coll.: Ta, Tampere, Santalahti, 5.6.1953 Y.M. (I). Die Äcidien befinden sich in helle, schliesslich rötliche Flecke an den Stengeln und der Unterseite der Blätter. Sie waren noch geschlossen, die Sporen (rundlich, 16-24 $\mu$ ) nicht völlig ausgebildet. Der Fundplatz ist nur etwa $50 \mathrm{~m}$ von der obenerwähnten Stelle mit Carex praecox entfernt. Später habe ich den Pilz jährlich an derselben Stelle beobachtet; das i. J. 1959 gesammelte Material wurde als Nr. 817 in Fungi Exsiccati Fennici verteilt.

9. P. iridis Rabh. - Auf*Iris spuria L. (I. halophila Ker-G.) und *I. violacea Sw. : Ab, Turku, Iso-Heikkilä, Botanischer Garten, 20.7.1953 Y.M. (II). Uredosporen $21.8 \times 26.9(20-23 \times 21-30) \mu$. KARI (1957, Nr. 416 und 417) sammelte den Pilz gleichfalls im Botanischen Garten auf $I$. germanica L. und I. halophila Pall. (I. gueldenstaedtiana Lepech.)

10. P. luzulae Lib. (P. oblongata (Link) Wint.). Auf Luzula pilosa (L.) Willd.: Ab, Turku, Ruissalo, Honkapirtti, 13.10.1951 Y.M. (II + III). P. luzulae weicht schon makroskopisch von $P$. obscura Schroet. ab, die an derselben Nährpflanzen (auch an L. pi- losa) schmarotzt und in Turku und dessen Umgebung nicht selten ist, aber nur ausnahmsweise Teleutosporen bildet. Die dunkelbraunroten Sporenanlagen befinden sich in intensiv dunkelroten Flecken, während sie bei $P$. obscura gelblichbraun sind und kaum deutliche Flecke hervorrufen. Teleutosporen $18.1 \times 59.5(17-20 \times 53-68) \mu$ (P. obscura, Abb. 1 a, $14-20 \times 35-50 \mu$; LiRo 1908 S. 216). Kennzeichnend für sie (Abb. 1 b) ist eine starke Verdickung

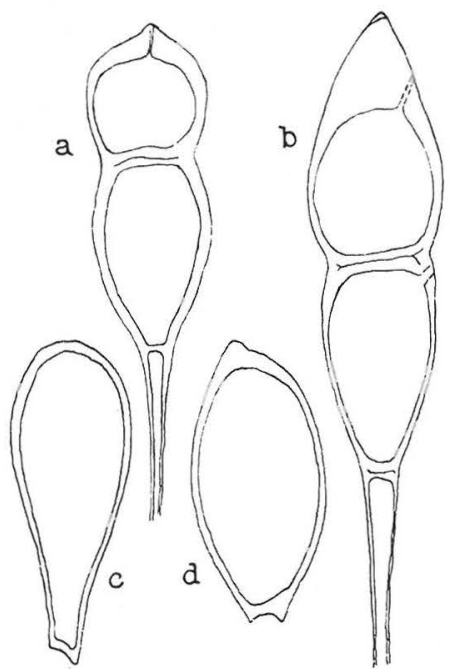

Abb. 1. a: Teleutospore von Puccinia obscura; b: desgl. von P. luzulae; c, d: Uredosporen von P. luzulae. (a: Ab, Kakskerta 1936, auf Luzula multiflora, Lauri E. Kari, Fungi Fenn. Exs. Nr. 436; b-d: Ab, Turku, Ruissalo 1951, auf Luzula pilosa, Y.M.) $750 \times$.

$(14-22 \mu)$ der Membran der oberen Zelle. Die untere Zelle ist bedeutend heller als die obere. Die Probe enthält verhältnismässig viele anomale Teleutosporen, deren untere Zelle entweder ganz unentwickelt oder unvollständig ausgebildet ist. Auch die Farbe der Sporen im ganzen ist viel heller als bei $P$. obscura. Die Uredosporen sind auffallend länglich, $17.9 \times 33.8(14-16 \times 28-38) \mu$ (Abb. 1 c, d). Im Pilzherbarium von TUR befindet sich eine unbeachtet gebliebene ältere Probe von $P$. luzulae, gesammelt von A. V. Auer am 12.9.1936 im Olhafen von Pansio in Turku. Diese Probe enthält nur Uredosporen, die $17.3 \times 30.3(15-18 \times 26-37) \mu$ messen. Ausserdem fand Auer den Pilz 1939 auch in Ruissalo (vgl. Rauhala 1959, S. 118).

11. P. polygoni-amphibii Pers. - Auf *Geranium pratense L.: Ab, Turku, Iso-Heikkilä, in der Nähe des Botanischen Gartens, 2.6.1953 Y.M. (I). Die Infektion war ziemlich schwach, nur etwa zehn 
befallene Blätter in dem relativ grossen Bestand. Der Pilz ruft kaum sichtbare rötlich-grünlich-gelbliche Flecke hervor. Äcidiosporen 13-18 $\times 17-21 \mu$, kantig-rundlich. Zellen der Pseudoperidie eckig, $18-30 \times 21-38 \mu$. Nach Hylander, Jørstad \& Nannfeldt (1953) und Jørstad \& Nannfeldt (1958) ist $P$. polygoni-amphibii früher nicht in Fennoskandien auf Geranium pratense angetroffen.

12. P. pulverulenta Grev. - Auf. * Epilobium hypericifolium Tausch: N, Helsinki, Botanischer Garten, 1922 Lauri E. Kari (II + III). Sehr starker Befall; Uredosporen $20.0 \times 22.2(17-23 \times 21-24) \mu$, Teleutosporen $18.3 \times 19.7(17-20 \times 25-32) \mu$, Die Wirtspflanze, die im Botanischen Garten als Unkraut auftritt, ist neu für einen Rostpilz in Fennoskandien.

13. P. singularis P. Magn. - Auf Anemone ranunculoides L. Bisherige Fundorte in Finnland: Ab, Piikkiö, Jauhosaari 26.5.1955 Y.M. \& Niina Tarén, 29.5.1956 Y.M., Niina Tarén \& Lisbeth Andersson (KARI 1957, Nr. 474), auch später jährlich beobachtet; Lohja, Torhola, 20.5.1962 Y.M., Ta, Lempäälä, Kuokkala, Moisio, 15.5.1936 S. Saarnijoki (Rauhala 1952), Lempäälä, Nurmi, etwa $250 \mathrm{~m}$ NW vom Gehöft Ansami, ein einziges befallenes Blatt, 10.5.1953 Y.M. Somero, Häntälä, 14.5.1955 Leo Lindgren (KARI 1957, Nr. 475). Ka, Viipuri, Lihaniemi, 21.6.1909 O. Brander (Rauhala 1952), Viipuri, Keihäsniemi, 30.5.1916 T. J. Hintikka (Rauhala 1952). - Lrro (1908, S. 258) erwähnt, dass das Myzel lokalisiert ist und keine Deformationen bei der Wirtspflanze verursacht. So dürfte es sich meistens auch verhalten, aber das Vorkommnis von Piikkiö macht eine Ausnahme. Dort trat der Pilz in den Jahren 1955 und

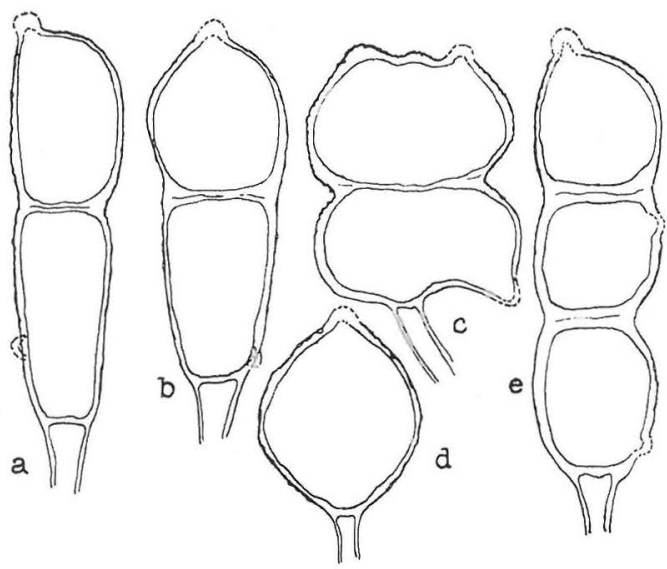

Abb. 2. Verschiedene Teleutosporenformen bei Puccinia singularis. $-750 \times$.
1956 ungeheuer reichlich auf, geradezu die Bestände des gelben Windröschens verheerend und oft schwere Missbildungen hervorrufend. Die Form der Teleutosporen ist sehr verschieden (Abb. 2 a, b, c), die Membran dunkelbraun. Ziemlich allgemein sind auch drei- oder einzellige Sporen (Abb. 2 e, d). Die durchschnittliche Grösse der Sporen beträgt $18-26 \times 32-45 \mu$. In meiner Probe aus Lempäälä sind die Sporen verhäıtnismässig regulär, gelblichbraun, $23-27 \mu$ breit und 39-48 $\mu$ lang; doch befinden sich darunter einige einzellige Sporen. Betreffs der Verbreitung der Art in Finnland hat RAuHALA (1952) die Auffassung geäussert, dass der Pilz in Westfinnland ein östlicher Einwanderer ist. Gerade in der Flora und Vegetation der Insel Jauhosaari in Piikkiö bemerkt man tatsächlich viele östliche Züge (vgl. Mäkinen \& TARÉn 1960, S. 128). Doch dürfte der Pilz sicher auch noch vielenorts in Südwest-Finnland zu finden sein.

14. Uromyces viciae-fabae (Pers.) Schroet. - Auf *Lathyrus palustris L.: Ab, Turku, Ruissalo, Kuuva, 11.9.1954 Y.M. (II+III). Uredosporen 19-23x $20-26 \mu$, die weningen Teleutosporen etwa $20 \times 26 \mu$. Der Pilz ist auf Lathyrus palustris aus Ostfennoskandien früher nur aus den Provinzen Kol und Kon in Ostkarelien bekannt (Liro 1908, S. 110).

15. U. lapponicus Lagerh. - Auf Oxytropis campestris (L.) DC. ssp. sordida. (Willd.) G. Hartm.: Li, Utsjoki, Tshuomasvaara, 11.7.1956 Y.M. \& Niina Tarén (I). In Ostfennoskandien früher nur aus den Provinzen Ks und Lps bekannt (Rauhala 1959, S. 159). Das Material wurde als Nr. 545 in Fungi Exsiccati Fennici verteilt.

16. U. lineolatus (Desm.) Schroet. (U. scirpi Burr.). - Die finnischen Fundorte sind die folgenden:Auf Hippuris vulgaris L.: Ab, Paimio, Viksberg, 2.8.1955 Ilkka Kukkonen (I) (KUKkoneN 1955).Auf Scirpus maritimus L. : Al, Lemland, Nåtö, 8.9.1956 Lauri E. Kari (II+*III; das Material wurde als Nr. 795 in Fungi Exsiccati Fennici verteilt). Ab, Parainen, Malmi, Bjukalö, 22.9.1951 Y.M. (II); Piikkiö, Harvaluoto, Ratsula, 11.8.1956 Y.M. (II+ III). N. Porvoo, 28.8.1937 J.I. Liro \& V.B. Lehtola (II, Roivainen 1953).

Auf diesen Pilz sollte besonders spät im Herbst ein Auge gefestigt werden. Beim Untersuchen der Proben von Scirpus maritimus im TUR erwies es sich, dass der Pilz wahrscheinlich zahlreiche Proben aus den Provinzen Al, Ab, N, Ka und St befallen hatte, aber nicht zur Sporenbildung gelangt war. Der Pilz tritt an den welkenden Blattspitzen auf, und bleibt wegen seiner Unauffälligkeit leicht unbeachtet. 


\section{ER $Y S I P H A C E A E$}

17. Erysiphe cichoracearum (Schlecht.) Salmon Auf *Hyoscyamus niger L.: Ab, Turku, Pansiontie, 1.10.1961 Y.M. \& Liisa Mäkinen. Eine einzige grosse und reich verästelte Pflanze, von der etwa 20 befallene Blätter gesammelt wurden. Nur Konidien.

18. E. fischeri Blumer - Auf $*$ Senecio viscosus L.: N, Hanko, Gåsörsudden, 4.10.1955 Y.M. Konidien und Perithecien, Durchmesser der letzteren 106$144 \mu$, im Mittel 128,4 $\mu$. E. fischeri ist in Finnland früher auf Senecio vulgaris L. (Al, N, lk, Sb) und auf S. silvaticus L. (Al) gefunden (TUR; KARI 1954, S. 23; 1957, Nr. 15; Rauhala 1957, S. 16).

19. E. graminis DG. - Auf *Poa pratensis L. ssp. alpigena (Fr.) Hiit.: Li, Utsjoki, Yläjalve, Kistuskaidi, 26.7.1956 Y.M. Nur Konidien, 11,6 $\times 24,8$ $(11-13 \times 23-29) \quad \mu$. Nach Rotvarnen (1958) ist der Pilz auf diesem Wirt allgemein. Rauhala (1957) führt ihn von $P$. pratensis aus $\mathrm{Ab}, \mathrm{N}$, St und $\mathrm{Ob}$ an; ich habe ihn auch in $\mathrm{Ta}$ (Somero, Kirchdorf 8.10.1961, nur Konidien) gesammelt.
20. E. martii Lév. - Auf * Lathyrus montanus Bernh. und *Lathyrus palustris L.: Ab, Turku, Ruissalo, Kuuva, 11.9.1954 Y.M. Nur Konidien. - Auf *Melilotus altissimus Thuill.: Ab, Uusikaupunki, Pietolanlahti, 16.8.1954 Y.M. (junge Perithecien und reichlich Konidien). KARI (1954, S. 33) erwähnt $E$. martii auf Melilotus altissimus nur aus der Provinze Kon in Ostkarelien. Blumer (1933) führt auf $M$. altissimus nur das Konidienstadium an, und auch die Angabe Karis bezieht sich auf Konidien.

21. E. montagnei Lév. - Auf * Cirsium oleraceum (L.) Scop.: Ta, Hämeenlinna, Ortelantie, 9.9.1957 Pekka Isoviita. Nach dem Finder war die Befallung des Cirsium oleraceum - Bestandes sehr stark. Der Durchmesser der in reichem Masse entwickelten Perithecien betrug $85-100 \mu$. E. montagnei ist von dem gleichfalls auf Cirsium oleraceum vorkommenden $E$. cichoracearum durch seine kleineren Perithecien und die geringere Zahl der Asci leicht trennbar. Auf Cirsium palustre ist E. montagnei bisher aus den Provinzen $\mathrm{Ab}$ und $\mathrm{Ta}$ bekannt (KARI 1954, S. 34). - Das Material wurde als Nr. 679 in Fungi Exsiccati Fennici verteilt.
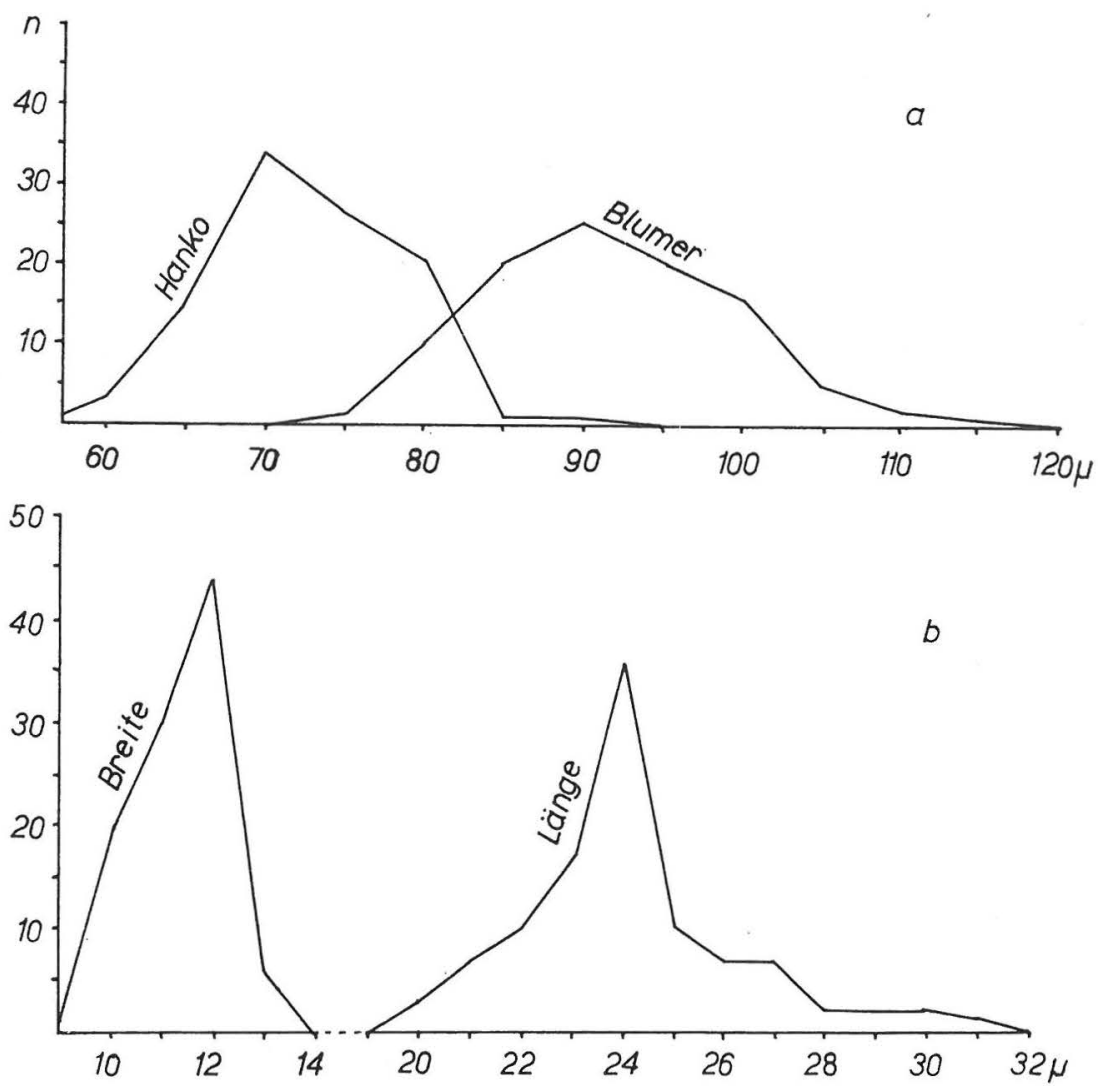

Abb. 3. Sphaerotheca euphorbiae. 1: Durchmesser der Perithecien nach BLumer und nach dem Material aus Hanko (1954); b: Grösse der Konidien im Material von Hanko. 
22. E. nitida (Wallr.) Rabh. - Auf * Thalictrum aquilegiifolium L. : Kb, Kitee, Närsäkkälä, Otravaara, nahe dem Gehöft Jaatinen, 5.8.1958 Y.M. (Perithecien und Konidien). Das Material wurde als Nr. 678 in Fungi Exsiccati Fennici verteilt.

23. * Sphaerotheca euphorbiae (Cast.) Salmon - Auf Euphorbia esula L.: N, Hanko, Gåsörsudden, 12.8. 1954 und 8.9.1955 Y.M. Die Wirtspflanze ist auf der Landenge Hankoniemi an mehreren Stellen als Rest der sowjetischen Besetzung zu finden. Der Pilz wurde trotz genauen Nachsuchens nur an der genannten Stelle gefunden, und auch alle von mir später angetroffenen Euphorbia esula -Bestände haben sich als rein erwiesen. Anscheinend hat sich die Art in Finnland nicht ausbreiten können und ist darum wohl nur als zufälliger Polemochor anzusehen. Wegen seiner durchgehenden Seltenheit möge er nachstehend etwas näher besprochen werden.
Sphaerotheca euphorbiae ist mit der amerikanischen S. mors-uvae nahe verwandt und hat wie auch diese ein dichtes sekundäres Luftmyzel, in dem die Perithecien eingebettet sind. Auch in den jetzt vorliegenden Proben ist das Luftmyzel sehr gut entwickelt, mit etwa 3-6 $\mu$ dicken Hyphen. Die reichlichen Perithecien mit schlect entwickelten Anhängseln sind aber auffallig klein, in der Probe von 1954 nur $60-88 \mu$, durchschen. $72.6 \mu$. und in der von 1955 durchschn. $79 \mu$.Die typischen Werte nach Blumer (1933) sind 75-115 $\mu$, durchschn. $91.9 \mu$, und nach SǍvulescu (1927) 83-100 $\mu$, durchschn. 91.2 $\mu$ (siehe Abb. 3 a). Diese grossen Differenzen haben mich veranlasst, die Perithecien an 18 Proben aus verschiedenen naturwissenschaftlichen Museen zu messen. Die Ergebnisse sind in Abb. 4 graphisch dargestellt; die Proben auf Euphorbia esula (einschl. E. virgata) sind durch offene, die auf anderen Wirtspflanzen (Euphorbia platyphyllos, E. helioscopia, E. palustris, E. dulcis) durch

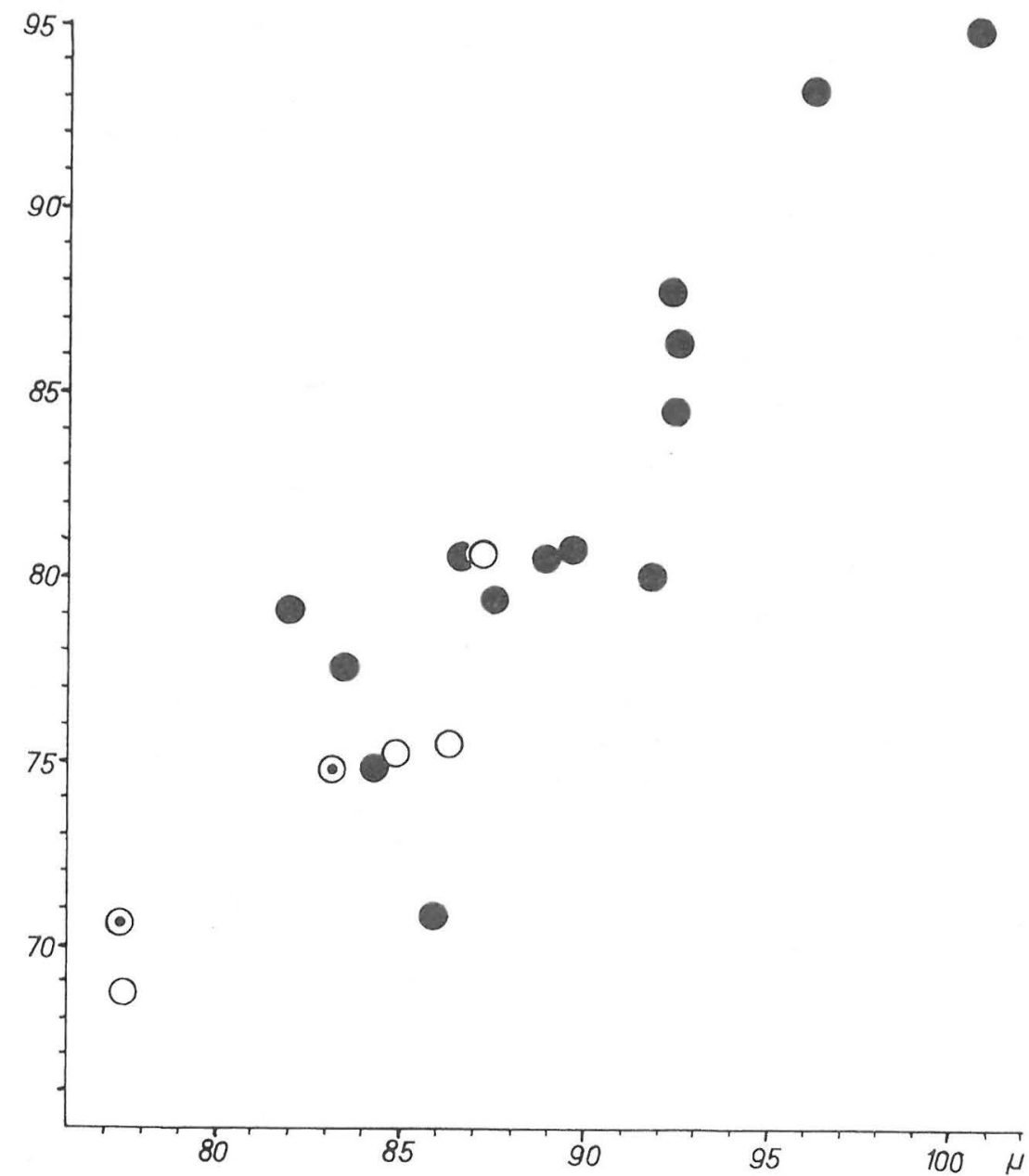

Abb. 4. Breite (Ordinate) und Länge (Ássisse) der Pecithecien von Sphaerotheca euphorbiae. Näheres im Text. 
gefüllte Kreise kenntlich gemacht. Es wurden Breite und Länge jedes Peritheciums gemesst. Es erwies sich, dass obschon die Perithecien in den meisten Proben bedeutend grösser als in den in Hanko gesammelten ( $\odot$ ) waren, es jedoch, besonders auf E. esula, doch auch auf anderen, auch solche mit kleinen Perithecien gab (Bucuresti 1920 auf E. esula $68.7 \times 77.6 \mu$ und $75.2 \times 84.9 \mu$; Pressburg 1889 auf E. palustris $70.7 \times 86.0 \mu$; Aubach 1938 auf E. dulcis $74.7 \times 84.3 \mu)$. Wahrscheinlich sind diese Formen mit kleinen Perithecien nur Ausdrücke einer klimatisch bedingten Phänovariation. Im allgemeinen ist eine grosse relative Lufteuchtigkeit förderlich für die vegetative Vermehrung, während geringe Feuchtigkeit und hohe Temperaturen die Bildung der Perithecien begünstigen. Nun regnete es sehr reichlich gerade um jene Tagen (12.8.1954 und 8.9.1955), als meine Proben gesammelt wurden: das Wetter also ungünstig für die Bildung der Perithecien. Dies dürfte wenigstens eine Ursache für die Kleinheit der Perithecien sein.

Die Asci sind in meinen Proben 45-60×66$111 \mu$, im Mittel $53.7 \times 89.4 \mu$ gross. Dies bedeutet, dass sie $15-20 \mu$ länger als die Perithecien waren! und gut mit Blumers (89.9 (80-100) $\mu$ und SàvULescus (87.8 (82-92) $\mu$ ) Werten übereinstimmen. In einigen Fällen war in den in Milchsäure erwärmten Präparaten festzustellen, wie der Ascus eingekrümmt im Perithecium lag und erst nach dessen Aufbrechen sich richtete. Die normale Grösse der Asci wird dadurch erklärt, dass sie im Perithecium vor äussere Einflüsse geschützt sind. Die Sporen sind $12.7 \times 20.0$ $(10-15 \times 15-24) \mu$, die Konidien (Abb. 3 b) $11.3 \times 24.0(9-13 \times 20-31) \mu$ (nach Blumer 10-14 $\times 22-27 \mu)$.

Im Fennoskandien ist Sphaerotheca euphorbiae mit Sicherheit früher nur zweimal gefunden. N. Moe (vgl. Jørstad 1925, S. 40) fand den Pilz 1872 im Botanischen Garten von Oslo auf Euphorbia palustris und 1939 sammelte Dr. I. Jørstad ihn auf derselben Wirtsplanze in Østre Baerum, Alv. (O, TUR).Ein Konidienstadium, das Buchwald (1941) zu dieser Art fügt, ist dazu in Dänemark auf Euphorbia cypa- rissias gefunden. Auch aus Estland kenne ich nur einen Fund, auf Euphorbia esula (»Tartumaa (Emed), in horto botanico Universitatis Tartuensis», 1937 E. Lepik; Fungi Estonici Exsiccati, Nr. 261). Dazu ist Oidium cyparissiae P. Syd. auf Euphorbia cyparissias in Schweden gefunden worden (Gästrikland: Gävle, Lövudden, $1943 \mathrm{~J}$. Nannfeldt; Fungi exsiccati suecici, Nr. 1484). Auch im ganzen Europa ist Sphaerotheca euphorbiae selten; so kennt z.B. BLumer (1933) den Pilz auf Euphorbia esula nur aus Kroatien und der Tschechoslowakei.

In den Konidienträgern (Probe vom 12.8.1954) schmarotzt Cicinnobolus cesatii De Bary, der in den Trägern braune Anschwellungen hervorruft und die Konidien zerstört.

Das Material wurde als Nr. 668 in Fungi Exsiccati Fennici verteilt.

24. S. fuliginea (Schlecht.) Salmon - Auf Erigeron canadensis L.: N, Hanko, Gåsörsudden, 12.8.1954 und 4.10.1955 Y.M.; Tenhola, Lappohja 3.10.1955 Y.M. In allen Proben reichlich Perithecien und weniger Konidien. Der Pilz wurde auf diesem Wirt in Finnland vordem nur einmal gefunden (1919, Helsinki; siehe Rauhala 1955). Das Material wurde als Nr. 674 in Fungi Exsiccati Fennici verteilt.

25. S. macularis (Wallr.) Jacz. - Auf *Potentilla thuringiaca Bernh. ssp. goldbachii (Rupr.) Aell.: Ta, Tampere, Vuohenoja, nahe der Haltestelle am Bahndamm, 6.8.1958 Y.M. Nur Konidien.

26. S. pannosa (Wallr.) Lév. - Auf * Rosa rugosa Thunb.: Ab, Piikkiö, Harvaluoto, 31.8.1955 Y.M. Konidien und Perithecien, deren Durchmesser etwa $89,5 \mu$.

27. Uncinula salicis (DC.) Winter - Auf Salix phylicifolia L.: Ab, Turku, Ruissalo, Choraeus, 30.9.1955 Y.M., Konidien und Perithecien. Auf diesem Wirte in Finnland nur einmal gesammelt (Ta, siehe Rauhala 1957, S. 24). 
Blumer, S., 1933: Die Erysiphaceen Mitteleuropas mit besonderer Berücksichtigung der Schweiz. - Beitr. zur Krypt. flora d. Schweiz, Zürich.

Buchwald, N. F., 1941: Mykologiske Smaating II. No. 3-6. - Bot. Tidsskr. 46, $422-429$.

Hylander, N., Jørstad, I. \& Nannfeldt, J. A., 1953: Enumeratio Uredinearum Scandinavicarum. - Opera Botanica $1: 1$.

Jørstad, I., 1925: The Erysiphaceae of Norway. Skr. Norsk. Vidensk. Akad. Oslo $\mathrm{I}: 10$.

$\longrightarrow$ - \& Nannfeldt, J. A., 1958: Additions and corrections to $\gg$ Enumeratio Uredinearum Scandinavicarum». Bot. Not, $111: 1,306-318$.

KARI, L. E. K., 1954: Beiträge zur Kenntnis der Erysiphaceen-Flora Finnlands. Ann. Univ. "Turku., A, XVII:3, $1-53$.

$\longrightarrow$ - 1957: Fungi Exsiccati Fennici. Fasc. I-X, No $1-500$. - Ann. Univ. Turku., A, II, 23, 1-194.

KukKonen, I., 1955: Uromyces scirpi (Cast.) Lgh. Paimiossa (V). - Luonnon Tutkija $59: 5,155$.

Liro, J. I., 1908: Uredineae Fennicae. - Bidr. Känned. Finl. Nat. o. Folk, 65.

Mäkinen, Y. \& Tarén, N., 1960: Piikkiön Harvaluodon ja Jauhosaaren kasvistosta. - Turun Ylioppilas VII, 93-128.
Rauhala, A., 1952: Puccinia singularis P. Magn neu für Finnland. - Arch. Soc. 'Vanamo' 7:1, 43-44.

$\longrightarrow$ — 1955: Maallemme uusia härmäsienten isäntäkäsveja. - Luonnon Tutkija 59, 29.

——— 1957: Kotimaisia härmäsienilöytöjä sekä tähänastiset tiedot härmäsienilajien kasvimaakunnittaisesta levinneisyydestä maassamme (Mehltaupilzfunde aus Finnland mit Berücksichtigung der bisherigen Verbreitungsangaben). - Karstenia IV, $14-26$.

$\longrightarrow$ 1959: Luettelo Suomen ruostesienistä ja tietoja niiden kasvimaakunnittaisesta levinneisyydestä Itä-Fennoskandiassa (Enumeratio Uredinearum Fennicarum et distributio hucusque cognita earum in provinciis phytogeographicis Fennoscandiae orientalis). - Kuopion Luonnon Ystäväin Yhdistyksen julkaisuja, B, $3: 3,1-181$.

Rorvainen, H., 1953: Mycotheca Fennica No 601900. - Institutum Phytopathologicum Universitatis Helsinkiensis.

-1- 1958: Poa rigens Hartm. - Pohjannurmikka. - Suuri Kasvikirja I, 405-407. Otava.

Sãvulfscu, Tr., 1927: Herbarium Mycologicum Romanicum, Fasc. I, No 17. 